\title{
On Time and Space in Hong Ke's Novels the Kalabu Sandstorm
}

\author{
Zhang Henan ${ }^{1, a}$, Teng Chunyan ${ }^{2, b}$ \\ ${ }^{1}$ College of Liberal Arts, Bohai University, Jinzhou, 121013, China \\ ${ }^{2}$ Colege of Foreign Languages, Bohai University, Jinzhou, 121013, China \\ a'Zhanghn1980@163.com, ${ }^{\mathrm{b}} 747121001 @ q q . c o m$
}

Keywords: the Kalabu Sandstorm; time; space

\begin{abstract}
Time and space is an important perspective to thoroughly interpret the theme in Hong Ke's novels. In his latest novel The Kalabu Sandstorm, Hong Ke still focuses on the theme of time and space. According to the temporality of natural scenery, Hong Ke highlights the value of natural aesthetic and the true meaning of life, which embodies the mental guidance of returning human beings to their spiritual homes. Also, Hong Ke emphasizes the narrative concept by destroying the traditional linear narrative time. Finally, using a variety of space juxtapositions, Hong Ke presents a persistent pursuit, exploration and closeness to the history and civilization of the western regions, and exhibits his anxiety and meditation over the barren culture consciousness of modern man.
\end{abstract}

\section{The Temporality of Natural Scenery}

The Kalabu Sandstorm is a great and well-known modern novel that is strongly rooted in the vast deserts of the frontier regions in the western part of China. The novel is permeated with rich romantic and idealistic sentiment in the Western Region. It also inherits and extends Hong Ke's writing device of the previous literary creation about the Western Region on love and life, humanity and nature. In the writer's eyes, many vivid literary images in the novel have become a carrier in the slow and natural flow of time. They are widely embedded in the chains of the plot of the story development, which present us a unique understanding of time formation and an exclusive forging of time dimension in Hong Ke's narrative time. Heidegger said: "Any understanding of the existence in the earth is bound to be in the field of time dimension [1]". The sense of time in The Kalabu Sandstorm is mainly reflected in the odd, grand and magnificent portrayals of natural images themselves, such as deserts, goblins, camels, sandstorms, swallows and so on. All of those natural sceneries with the primitive elements entail a mysterious and ancient legendary, which are distributed widely in each part of the story development. Those natural sceneries seem to convey a deep and profound sense of history and civilization in the novel like a tunnel of a long history connecting the ancient time to the present time. The temporal characteristics of these natural images used in the novel are mainly reflected in the following several aspects:

In order to create a desolated and forsaken geographical space, the writer lays stress on the detailed descriptions and narrations of the natural time dimension in the Western Region. This kind of writing technique makes the eternity of time by far more prominent and distinctive. Tracing the history of the development of human civilization, the desert is generally regarded as an eternal existence as well as a huge reference of human survival. On one hand, the desert is endowed with the natural features of seasonal cycle or solar terms. On the other hand, the desert contains a large number of humanistic ideals that the writer has been pursuing and devoted to for many years. In other words, the desert "belongs to the art palace of the world without boundaries, and it is represented before us like a silent and soundless language that cannot be expressed with words. After many centuries of the baptism of the nature, they are still well preserved till today unchangeably [2]". In spring, the vast desert and sky seem to fuse together. Whatever one strains one's eyes to look afar, he cannot distinguish between heaven and earth. In summer, a dune is a big warm nest which is like a burning flame. During the day, the rolling sun on the western horizon 
shines a little brighter, and it filters on the smooth sands through the holes of sandstone. When night falls, the desert becomes a sea of moonlight. Edward Abi, an American writer, wrote in Desert Solitaire: "there are some things in the desert that we human beings cannot understand. It is true that there are things in the desert that lack in the mountains and the sea, no matter how tall they are or how old they are [2]". In my opinion, something unique to the desert is not only that primitive and vigorous power, but also the character and morality that are injected in the spiritual world of modern man which has been lacking in them. Zhang Ziyu deeply loves the desert, and the desert gives him clarity and loyalty like the son of nature: If one really loves a person, he should love her or him unreservedly, just like the desert. Zhang Ziyu didn't come to realize that love is without reservation until he arrived deep in the desert. In the description of story development, Zhang Ziyu sang the Kazak minority's folk song "Swallow" in the desert, walking through the middle of the desert. During the period both his character and spirit mix with a flavor of the desert that is possessed with boundlessness, broad natural disposition as an organic whole, and eventually become a whistling arrow of love that shot into Ye Liya's heart. However, the male protagonist Meng Kai in the novel is a man of disappointment of love affairs. Since he broke up with his beloved person, Meng Kai has been placed his inquiry of love, the exploration of value of life and interpretation of deserts into an imaginative, fantastic and grotesque world. Although the deserts mercilessly take away her beloved person, they give him the mind of forgiveness and the heart of moral. For instance, when the Kalabu sandstorm drew to a close, Meng Kai bravely rescued his past rival in love Zhang Ziyu and sent him back to Ye Liya. This is also the climax of the story development, and paves the way for Meng Kai to clear up his doubts and enhance the realm of life. The entire novel from beginning to end thoroughly echoes the footfalls of humanitarian redemption. The emotional entanglements of Meng Kai, Zhang Ziyu and Ye Liya intertwine with the natural sceneries of the desert. Facing with the magnificent nature, the illness and wickedness of human insignificance and pettiness can be cured and eliminated radically. The eternal life that is unique to the desert is promoted to a level where benevolent love becomes eternal in drawing human mental maps. The characters of the novels have been thinking deeply about lose and possession, hostile and benevolent love in life. It is the permanence of the desert that is mending the vulnerability of modern man's spirit and instructs them how to contemplate the true meaning of life: "The desert didn't say anything about the world or life. It is completely passive and never has the initiative to do anything. As usual, the desert is lying there, quiet and silent, like a bare skeleton, simple, desolate, plain and completely valueless, and so the desert simply can not arouse people's passion for life except meditation [2]".

In terms of the philosophical idea of ecological integrity, the novel weaves a network of the natural image and human fate of survival, causing natural scenery to form a cycle of time. In the Kalabu Sandstorm, the cycle of time of all living things in nature that is always surging in nature mainly refers to the life divine of the endless blending of every living creature in their surroundings. Holmes said: "The natural things have been beyond the nature of human domestication and human experience, and finally they get their own life in the natural world [3]". The novel highlights a comfortable life in the process of birth of life by imagining and narrating the goblin in the deep desert: If the livestock, male camel's "water for life" spurts on the roots of Nitraria sibirica in the desert, Cynomorium will grow; If male camel's "water for life" spurts on the roots of Haloxylon ammodendron and Tamarix ramosissima, Cistanche salas will be formed; if Cynomorium songaricum and Cistanche salas associate together, the goblins will grow. As is known to all, the goblin has a very high medical value. Once humankind consumes them, they will at once surge a wonderful and magical power in their body which generate the next offspring. In fact, it is the natural forces which are poured into the human body that multiply and breed a new life. "The natural scenery and their lives flourish in the process of the eternal life of human growth from birth to death. Each kind of life is represented by its unique way of life to support all the other things [3]". For instance, some unique plants in the desert such as the vast Gobi desert, the golden camel, swallows of a meteor, camel of God, the goblins, Haloxylon ammodendron and Tamarix live harmoniously with those characters who has the features of spirit-orientated natural sons such as 
Zhang Ziyu, the camel man, the Mongolian, and the old teachers. These creatures in the desert comply with the law of Nature to form a common survival circle and symbiont system. Sometime, life and death constitutes an eternal cycle of time: the wind blows the dead swallows to the Ebinur Lake, and they soon become the fish; the camels like flame died in the desert, and they become Yadang's swallow and spread their wings to fly. It is the form of this blend of natural things that keep the ecological balance of nature and coexist. Whether animal, plant or human, they have a tough action to complete the projection of natural circulation time in the process of human history. In addition, the writer in the novel also intends to set up a unique natural landscape in Xinjiang, the Kalabu sandstorm, making it appear frequently throughout the whole story, "The Kalabu sandstorm brings along snow and ice in winter and sand and stone in summer. Wherever it arrives, the earth becomes Yadang, and birds break their wings and die. Populus diversifolia becomes the walking camels, while others fall into love with their beloved ones.” The Kalabu Sandstorm is not only served as the unique background of story development, but also internalized the driving force for the emotional development of the characters every time it falls. Not only did the Kalabu Sandstorm play a crucial part in examining the true love of Zhang Ziyu and Ye Liya, but also comfort and heal the wounded heart of Meng Kai. Similarly, the Kazak minority's folk song "Swallow” is also frequently reciprocating in the intensive part of the novel compared with others. From the beginning of Sven Hedin's expedition in the Inner Asia from a hundred years ago up to now, the writer shows that "Swallow" unquestionably becomes the main melody of the works, which will be sung merrily all the way from the west region to Xi'an along the Silk Road. In the novel, the swallow, "The desert spirit", intertwined with the ancient song "Swallow", combining-each other closely and intimately. They are seen as not only historical and cultural performances, but also have become symbols of the characters' spirit. What makes us amazed is that the circulation of "Swallow" bridges the gap and loss of reading boundary due to Hong Ke's desultory narrative, and continuously gives the readers a sense of grandness and wholeness.

\section{The Prominent Subjectivity of Narrative Time}

For a novelist, time is an immense maze. It means that time creates the history of mankind, establishes handed-down performances, and also collects the remains of the ancient times. From the perspective of narrative theory of modern fiction, the method of narrative time such as delay, dispersion, frequency and distance profoundly shaped the characteristics and nature of novel as well as constantly polishing the writer's writing styles. Frank once said: “The sound of the ticking clock can be seen as a model of plot in a novel, and even be considered a sort of arrangement by giving time a form or making the time personal. The gap between the two sounds is filled with a meaningful process. The realistic nature of time and the careful arrangement of the two sounds of the ticking clock are the attributes of the modern novels...... The history of novel shows that a novelist is more and more concerned about this sort of arrangement as well as the fun of diminishing the time and the reality of the balance [4]”. In the Kalabu Sandstorm, Hong Ke began experiencing the subject of time, and separates the narrative time and the chronicle of story. Through breaking the chronicle of novel, using flashback formed by stream of consciousness and narration interposed with memories attributes; Hong Ke makes the novel truly experience the epic of time in the aesthetic gaze by way of expression of non-linear narrative time.

The first section of the Kalabu Sandstorm basically determine the trend of story development: the day before school holiday, Ye Liya, a girl in Jinghe, Xinjiang, decided to broke up with her boyfriend Meng Kai with whom she has falled in love for years and chose to disappear together with Zhang Ziyu in the desert after she heard that gentlemen singing the folk song of Kazak minority “Swallow”. From the readers' points of view, Hong Ke should immediately deduct and lay out the emotional entanglements among the three characters Meng Kai, Ye Liya and Zhang Ziyu following the beginning of plot in the story. On the contrary, the writer intends to set aside the key clue, and does not give the readers any buffer time for breathing or thinking about the following story developments. Instead, he is at will to turn the narrative sights from the reality of characters' life into a situation that dates back to the past. He began to insert a large number of contents that 
seemed irrelevant to the novel, such as some descriptions of plants in the desert, Sven Hedin's adventures and his love stories, the boyhood of Meng Kai and Wu Ming Sheng, the college times of Meng Kai and Zhang Ziyu. Hence, by applying flashbacks and insertions in the narrative time, the writer breaks the perception of inertia caused by the continuity of time, and constructs a wholeness of narrative time by non-causal relationship deduction and non-linear time. In the changes and restructure of time, the novel hides the logic emotional relationship between Meng Kai, Ye Liya and Zhang Ziyu, but highlights the unique values of natural aesthetic that the curve of time brings about. As a whole, the delicate application of this kind of narrative time into the novel presents us the mental state, the process of wound healing and unfading recollections of growth after Meng Kai were disappointed in love affairs.

In those insertions of fragments, the novel shows that the stream of consciousness of Meng Kai who is indulge in fantasy but negligent of action by using a gentle and mild narration rhythm. Through the winding and diffuse of consciousness "changed the time structure of the story, disrupted and reorganized the order of time with free association. Time suddenly turned to the narrative, sometimes going back down, then fell back, and then pauses, forward, and backward in narrative time...... [5]". In the novel, the time order of Meng Kai's mental activity is the main criterion of narrative time in the novel structure of the writer. The narration changes from the narrative of Ye Liya to the description and narration of Xinjiang minority people's way of living, which are imbued with lives of the people in the ethnic minority on the edge of regions: the Kazak minority's wedding customs, the old and ancient ballads and the camel man, the Mongolia grassland singer; the vast expanse of the Gobi desert and the birch forest nearby at the outskirts of oasis, Chinese wolfberry like red agate, sweaty and juicy watermelon, Uighur baked pit Nang, the koje, the leghman, pilaf, etc. A large amount of these symbols of the life are pouring into the narrative. The moment the readers who temporarily forgot the characters' feeling was immersed in the life of ocean in the western regions, the writer draws their attention to the Europe ancient proverb, myths, poetry and epic, such as Paris, Menelaus, "Leili and Majnoon”, “The Trojan War”, and "Traveling Story in the Inner Asian" and so on. This free of innovative ideas based on a particular culture, legends and anecdotes, history into account, transfer high speed, expands the capacity of the story and create an artistic atmosphere linking to ancient and modern, through Chinese and Western.

The control and layout of narrative time is also reflected in the retrospective narration as the narrator recalls the days in the past: the narrative time unconsciously detaches from the real world and leaps into a point where Zhang Ziyu received a key university's notice in Xi'an province in 1987. At the moment, Zhang Ziyu's growth experience came into the readers' vision. A youth from the countryside came to the desert, which was unbearable to the anxieties, restlessness and impatience of the city life. It was magical that the desert cured Zhang Ziyu's spirit illness that was accumulated over the years, and relieve his burden and confusion over the city's life. In the descriptions of Meng Kai's goblin business, the novel guides readers into a sort of memory which pointed to the family legendary between $\mathrm{Wu}$ Mingsheng, the playmate in the childhood and his father, brother, and grandfather. Hong Ke mainly focuses on the stage of person's life from 1959 to 1961. Shu Hezhe, a well-known scholar, in his masterpiece, Wandering Goddess of Memory, cited that that memory is not just an abstract or merely personal thing, but it is defined as reviewing the past of nation, make it fuse into it and change one's fate. Take a section in the novel for example, the shortage of food that natural disaster of the three years brought about caused Meng Kai in Xinjiang insight into "low standard" and "vegetables generation" that Shaanxi people had experienced. As a result, there formed a bright contrast between Shaanxi people's hunger for food and Xinjiang people's rich reserves of grain. In the portray of the Chinese special memory, the writers lay out the chapter in which Li Yun and Zhang Ziyu in the college times visited Mu Us Desert. The kind of the plot about memories is scattered. The features of narrative time in the whole novel are characterized by flashback. The narrative clues are intricate and continuously interlaced, so that the readers formed a seemingly free but with actually firm reading experience. 


\section{The Juxtaposition of Narrative Space}

Bakhtin has stressed: "in the art of space and time of the literature, space and time often integrate together in a specific understanding of the overall. Time is condensed and shortened into the art of visible things; yet space is the trend of tension, which was involved in the movement of the time, the plot, the history [5]". In my opinion, the Kalabu sandstorm consciously deforms the narrative time to make it shorten, extend or delay in the process of story development, and pursue a kind of subjective and irruptive effect of time experience. In addition, the space is marked as the core qualities of constructing the plot of the story in the literary works. The novel establishes a necessary relationship between the symbolic space and the characters and plot so that it weaves a unified, integrated and orderly world. This kind of space is mainly reflected in multiple juxtapositions of space meaning in the novel.

Juxtaposition is defined as an important creation concept of criticism in modern fiction study originially proposed by a famous philosopher Joseph, and gradually becomes one of the most important and significant expressions of spatial meaning in the literary works. Joseph pointed out that juxtaposition refers to those small or large units of meaning placed side by side, so that the unity of text does not exist in time, but in space [5]". From the aspect of the structure of the novel, the Kalabu Sandstorm breaks the causal relationship in the story narrative, and even those images, myths, folklores, hints and symbols are placed in space so as to comprise a distinctive artistic style of modern creation.

Since the creation of the Beautiful Shepherd, the space has been the theme of Hong Ke's novels that he cannot avoid. From then on Hong Ke kept composing plenty of novels on the geographical space that he was very familiar with, including Weibei, Xi'an, Qinghai, Northern Frontier, and Southern Frontier. Above all, the space of Hong Ke's creation extends from Tianshan, Ertix River to Artai Mountain. His traces of creation extend to those minority living areas of the Western Region such as Hui, Mongolian, Kazak and Uygur minority. As a representative of Shanxi people, Hong Ke has been amazed and shocked at the magnificent beauty of Xinjiang. In the novel, his main characters are generally divided into different spaces with regional attributes: The space constituted by Shanxi people includes "Li Yun and Zhang Ziyu”, "Tao Yaling and the students who learn traditional Chinese medicine", "the production team in Zhi Hezi and the kids", "Wu Mingsheng, his father and grandfather", "Zhang Ziyu, his father and grandfather"; The space constituted by Xinjiang people includes "Ye Liya and Meng Kai", "Meng Kai and the driver cousin”, "Meng Kai and his uncle in Jinghe”, "Meng Kai and his parents in Tacheng”, "stockman in the pasture", "the geography teacher and Chinese teacher"; The space constituted by Shanxi and Xinjiang people includes "Meng Kai, Wu Ming and their university students", "Meng Kai and Tao Ya Ling". In the constantly changes of living space of these characters, the logical order of the narrative time has lost its essential significance, but highlights the process of enculturation and fusion of cultural. Above all, much attention is paid to the juxtapositions of historical and realistic space with cultural attributes. For instance, the Desert Research Institute, Shi Hezi team, Peng Jiamu and Yu Chunshun constitute a living space in reality; The historical and cultural space in Central Asia consists of the Greek War of Troy, Homa Epic, Germanic epic, Nibelungenlied, Beowulf, The Garden in the great ancient Persia, Orchard and Sven Hedin's Travelling in the Inner Asia. The ethnic historical and cultural space in Xinjiang consists of Genghis Khan, a Mongolia leader, Kazakh folk song "Swallow" and so on. Chinese historical and cultural space includes Chinese classical masterpiece "A Dream of Red Mansions", "Chinese New Culture Movement on May 4th", "the Power of Romantic” that was standard for Lu Xun's passion”, "the Spirit of Toni”, "the article and the poem of Wei and Jin Dynasties that Liu Bannong offered to Lu Xun by way of appreciation”; “the Story of Qin Qiong's selling horses and Yang Zhi's selling knives”, etc. These juxtaposition of various spaces showed that the attention of Hong Ke is paid to the hidden and mysterious cultural characteristics of different spaces in the Western Region. Through the uses of various juxtapositions of cultural space units, the novel has really created and reproduced an unprecedented, grandiose and strong cultural atmosphere. By using juxtapositions of space, Hong Ke makes the supremacy of the world culture vividly portrayed and demonstrated. 


\section{Acknowledgement}

This work is one of the achievements in the research of ecological novels in the new century, which is based on the doctoral program of Bohai University.

\section{References}

[1] Martin Heidegger. Existence and Time [M]. Chen Jiaying, Wang Qingjie, trans. Shanghai: Shanghai Sanlian Press, 1999:1.

[2] Edward Abbey. Desert Solitaire [M]. Li Rui, Wang Yansheng, trans. Haikou: Hainan Publishing House. 2003: 119, 279, 276.

[3] Holmes Rolston. Philosophy into the Wilderness [M]. Liu Er, Ye Ping, trans. Jilin: Jilin People’s publishing House, 2003: 209, 9.

[4] Frank Kermode. The Space Form of Modern Fiction [M]. Liu Jianhua, trans. Liaoning: Liaoning Education Press, University of Oxford Press, 2000: 52-53.

[5] Ma Dakang, Ye Shixiang. A Study on Literary Time [M]. Beijing: China Social Science Press, 2008: 229, 271, 171. 